\title{
Some variables affecting the characteristics of Eudragit E-sodium alginate polyelectrolyte complex as a tablet matrix for diltiazem hydrochloride
}

\author{
REHAB MOHAMMAD YUSIF* \\ IRHAN IBRAHIM ABU HASHIM \\ MARWA SALAH EL-DAHAN \\ Department of Pharmaceutics, \\ Faculty of Pharmacy, \\ Mansoura University, \\ Mansoura, 35516, Egypt
}

Accepted December 3, 2013

\begin{abstract}
Eudragit E (EE)-sodium alginate (SA) polyelectrolyte complexes (PECs) were prepared at $\mathrm{pH} 4$ and 5.8 using sodium alginate of high (SAH) and low viscosity (SAL). The optimum EE-SA complexation mass ratio was determined using viscosity measurements. Interactions between EE and SA in PECs were characterized by Fourier transform infra-red spectroscopy (FT-IR) and differential scanning calorimetry (DSC). Diltiazem hydrochloride (DTZ $\mathrm{HCl}$ ) tablets were prepared using the prepared EE-SA PECs and their physical mixtures at different ratios as matrices. Tablets were evaluated for swelling characteristics and in vitro drug release. Tablets containing EE-SAH physical mixtures of ratios (1.5:1 and 1:3) as matrices were effective in achieving sustained release of DTZ $\mathrm{HCl}$, where the percent drug released was significantly $(p<0.05)$ decreased compared to that from tablets either containing the same ratios of EE-SAL physical mixtures or the preformed EE-SAH and EE-SAL PECs.
\end{abstract}

Keywords: polyelectrolyte complex, diltiazem hydrochloride, Eudragit E 100, sodium alginate, viscosity, tablet matrix

Modification of polymer properties can be achieved by copolymerization or derivatization, a strategy that was successfully applied in the past. However, this approach has the major drawback that new chemical entities are introduced with an unknown toxicological profile. Before these products can be evaluated in animals and also in human clinical trials, a lot of time and resources must be spent to assess their safety. To overcome this problem, physical modification of the polymer, rather than chemical, is a sound approach. In this respect, polyelectrolyte complexes (PECs) may provide a valuable tool to design drug delivery systems with specific physicochemical properties. PECs obtained

\footnotetext{
* Correspondence; e-mail: rehabyusif@yahoo.com
} 
R. M. Yusif et al.: Some variables affecting the characteristics of Eudragit E-sodium alginate polyelectrolyte complex as a tablet matrix for diltiazem hydrochloride, Acta Pharm. 64 (2014) 89-104.

as precipitates on mixing cationic with anionic polymers in aqueous solutions have been reported previously. The advantages of using PECs as polymeric carriers in controlled systems for drug release were already investigated (1).

It is evident that the properties of PEC depend on various factors such as nature, position of ionic groups and molecular mass of the polymers (2-4). Other factors influencing PEC properties include $\mathrm{pH}$ of the solution, temperature and sometimes the order of mixing (5). PECs can be obtained by simultaneous dissolving of polymers in a suitable solvent, followed by solvent evaporation or by template polymerization. Interaction between the two oppositely charged polymers must occur in an environment of a suitable $\mathrm{pH}$ where a significant degree of polymer ionization exists (6). Accordingly, the possibility of interaction between physical mixtures of polymers within a tablet matrix could occur in dependence on the $\mathrm{pH}$ of the dissolution media, thus forming an in situ PEC (7).

Eudragit E is a cationic copolymer of 2-dimethylaminoethyl methacrylate, methyl methacrylate and $n$-butyl methacrylate, with the mean relative molecular mass of about 150,000 . The ratio of dimethylaminoethyl methacrylate groups to butyl methacrylate and methyl methacrylate groups is about 2:1:1. The content of dimethylaminoethyl groups is between 20.8 and $25.5 \%$ (8).

A natural substance, sodium alginate (SA), has received much attention as a vehicle for controlled drug delivery. It is found in brown seaweed and algae (9). It belongs to polysaccharides composed of $\alpha$-L-guluronic acid and $\beta$-D-mannuronic acid residues, arranged in homopolymeric blocks of each type and in heteropolymeric blocks. It was reported that the alginate monomer composition had a major impact on the drug release properties of different formulation systems (10). Furthermore, alginate particle size, viscosity and concentration affected not only the drug release rate but also the release mechanism (11).

Diltiazem hydrochloride (DTZ) is a coronary and peripheral vasodilator generally indicated for the treatment of angina and hypertension. Conventional tablets containing DTZ $\mathrm{HCl}$ are administered 3 or 4 times daily due to its short biological half-life of 3 to 5 h. These properties make it a suitable candidate for a controlled release formulation (12).

In recent years, great interest in the formation and characterization of Eudragit $\mathrm{E}$ PECs has been directed towards controlled release applications. In this regard, different polyanions of natural and synthetic origin were used as oppositely charged polyelectrolytes in complexation with Eudragit E (13-16).

Thus, the purpose of this study was to investigate the effect of the $\mathrm{pH}$ of complexation and sodium alginate viscosity on the interaction ratio of Eudragit $\mathrm{E}$ with sodium alginate and to characterize the products formed using FT-IR and DSC techniques. Also, to compare the tablet formulations composed of physical mixtures of Eudragit E and sodium alginate with those made of the prepared PECs. The comparison was made in terms of swelling characteristics and drug release under simulated gastric conditions using diltiazem hydrochloride as a model drug. Finally, the possibility of polymer-polymer or drug-polymer interaction within tablet matrix during the drug release process was studied. 
R. M. Yusif et al.: Some variables affecting the characteristics of Eudragit E-sodium alginate polyelectrolyte complex as a tablet matrix for diltiazem hydrochloride, Acta Pharm. 64 (2014) 89-104.

EXPERIMENTAL

\section{Materials}

Eudragit E-100 (EE) was obtained from Röhm Pharma, Darmstadt, Germany. Diltiazem hydrochloride was kindly supplied by Egyptian Int. Pharmaceutical Industries Co. (10 th Ramadan City, Egypt). Sodium alginate of high viscosity (SAH), viscosity of $2 \%$ solution at $25^{\circ} \mathrm{C} 14000 \mathrm{mPa}$ and sodium alginate of low viscosity (SAL), viscosity of $2 \%$ solution at $25^{\circ} \mathrm{C} 100-300 \mathrm{mPa}$ s, (Sigma, USA). Potassium bromide for Fourier transform infrared (FTIR) spectroscopy (UVasol ${ }^{\circledR}$, Merck, Darmstadt, Germany). Other materials were of analytical grade.

\section{Viscosity measurements}

Solutions of EE, SAH, SAL in acetic acid/sodium acetate buffer at $\mathrm{pH} 4$ and 5.8 were prepared. Eudragit E solutions were added to either SAH or SAL solutions in different ratios to make $50 \mathrm{~mL}$. The mixtures were shaken for $1 \mathrm{~h}$, followed by centrifugation at $5000 \mathrm{rpm}$ for $20 \mathrm{~min}$. Supernatants were collected and viscosity was measured using a rotational viscometer, Haake, Rotovisco RV 3, MV I sensor system (Berlin, Germany) at a shear rate of $150 \mathrm{~s}^{-1}$. The results of viscosity at different $\mathrm{pHs}$ were expressed as the mean of three determinations.

\section{Preparation of PECs}

Mixtures of polymer solutions that showed the lowest viscosity at $\mathrm{pH} 4$ and 5.8 were used in the preparation of PECs. Polymer solutions were mixed together and the produced precipitates were washed twice with distilled water to remove any free polymers, then dried under vacuum at $40{ }^{\circ} \mathrm{C}$ to constant weight. Then, the dried complexes were ground into powder using a mortar and pestle, passed through a $200 \mu \mathrm{m}$ sieve and then stored in tightly closed glass-bottles for further studies.

\section{Study of polymer-polymer and drug-polymer interactions}

Infra-red spectra of EE, SAH, SAL, the prepared PECs at $\mathrm{pH} 4$ and 5.8 in addition to their physical mixtures were determined using a Fourier transform infrared spectrophotometer (Thermo Fisher Scientific, Inc., Waltham, MA, USA). Each sample (2 mg) was mixed with $200 \mathrm{mg}$ of potassium bromide $(\mathrm{KBr})$. These mixtures were ground into fine powder and then compressed into $\mathrm{KBr}$ discs using a hydraulic press. Each $\mathrm{KBr}$ disc was scanned over a wave number region of $500-4000 \mathrm{~cm}^{-1}$ and the resolution was $4 \mathrm{~cm}^{-1}$. Characteristic bands were determined for all samples.

The powders were further analyzed using differential scanning calorimetry (Pyris 6 DSC, Perkin Elmer, USA). Temperature calibration was performed using indium as a standard. Samples $(4 \mathrm{mg})$ were weighed directly in aluminum pans, which were then closed. The samples were kept for $1 \mathrm{~min}$ at $30{ }^{\circ} \mathrm{C}$, then heated from 30 to $400{ }^{\circ} \mathrm{C}$ at a heating rate of $10{ }^{\circ} \mathrm{C} \mathrm{min}-1$ under constant purging with dry nitrogen at $30 \mathrm{~mL} \mathrm{~min}-1$. Finally, they were kept for $1 \mathrm{~min}$ at $400{ }^{\circ} \mathrm{C}$. 
R. M. Yusif et al.: Some variables affecting the characteristics of Eudragit E-sodium alginate polyelectrolyte complex as a tablet matrix for diltiazem hydrochloride, Acta Pharm. 64 (2014) 89-104.

The possibility of polymer-polymer or drug-polymer interaction during the drug release process was also studied according to the procedure described by Bani Jaber et al. (15). EE-SAH or EE-SAL physical mixtures of ratio (1.5:1) were kneaded with $0.1 \mathrm{~mol} \mathrm{~L}^{-1}$ $\mathrm{HCl}$ or phosphate buffer of $\mathrm{pH}$ 6.8. Also, drug-polymer interaction was studied by kneading physical mixtures of DTZ HCl $(1 \mathrm{~g})$ and each polymer $(2 \mathrm{~g})$ using $0.1 \mathrm{~mol} \mathrm{~L}^{-1} \mathrm{HCl}$ or phosphate buffer of $\mathrm{pH} 6.8$ with subsequent drying and grinding into powders. Kneaded polymer-polymer or drug-polymer powders were run for FT-IR and DSC analysis, in comparison with the pure drug, untreated polymer-polymer and drug-polymer physical mixtures.

\section{Preparation of tablets}

The formulations studied are shown in Table I. Powders were manually mixed in a plastic container for $10 \mathrm{~min}$ with DTZ $\mathrm{HCl}$ and magnesium stearate $(1 \%, \mathrm{~m} / \mathrm{m}$ as a lubricant) to make $275 \mathrm{mg}$ tablets. Tablets were obtained by direct compression using a single punch tablet machine (Type EKO, Erweka Apparatebau, GmbH, Germany) in $10 \mathrm{~mm}$ of die. The compression pressure was adjusted to give tablet hardness between 71-78 N. Tablets were found acceptable for friability $(<1 \%)$.

Table I. Tablet formulations studied

\begin{tabular}{|c|c|c|c|c|c|c|c|c|}
\hline \multirow[b]{2}{*}{ Formula $^{a}$} & \multirow[b]{2}{*}{ Code } & \multicolumn{7}{|c|}{ The quantity of ingredients in tablet (mg) } \\
\hline & & EE & SAH & SAL & $\begin{array}{c}\text { EE-SAH } \\
\text { PEC at } \\
\text { pH } 4\end{array}$ & $\begin{array}{c}\text { EE-SAH } \\
\text { PEC at } \\
\text { pH } 5.8\end{array}$ & $\begin{array}{c}\text { EE-SAL } \\
\text { PEC at } \\
\text { pH } 4\end{array}$ & $\begin{array}{l}\text { EE-SAL } \\
\text { PEC at } \\
\text { pH } 5.8\end{array}$ \\
\hline Eudragit E & $\mathrm{EE}$ & 182 & & & & & & \\
\hline $\begin{array}{l}\text { Sodium alginate of high } \\
\text { viscosity (SAH) }\end{array}$ & SAH & & 182 & & & & & \\
\hline $\begin{array}{l}\text { Sodium alginate of low } \\
\text { viscosity (SAL) }\end{array}$ & SAL & & & 182 & & & & \\
\hline EE-SAH ph. mix. 3:1 & PMH1 & 136.5 & 45.5 & & & & & \\
\hline EE-SAH ph. mix. $1.5: 1$ & $\mathrm{PMH} 2$ & 109 & 73 & & & & & \\
\hline EE-SAH ph. mix. 1:3 & PMH3 & 45.5 & 136.5 & & & & & \\
\hline EE-SAH PEC at $\mathrm{pH} 4$ & PCH1 & & & & 182 & & & \\
\hline EE-SAH PEC at pH 5.8 & $\mathrm{PCH} 2$ & & & & & 182 & & \\
\hline EE- SAL ph.mix. 3:1 & PML1 & 136.5 & & 45.5 & & & & \\
\hline EE- SAL ph.mix. 1.5:1 & PML2 & 109 & & 73 & & & & \\
\hline EE- SAL ph.mix. 1:3 & PML3 & 45.5 & & 136.5 & & & & \\
\hline EE-SAL PEC at $\mathrm{pH} 4$ & PCL1 & & & & & & 182 & \\
\hline EE-SALPEC at $\mathrm{pH} 5.8$ & PCL2 & & & & & & & 182 \\
\hline
\end{tabular}

a Each formula contains $90 \mathrm{mg} \mathrm{DTZ} \mathrm{HCl}$ and $1 \%$ magnesium stearate. 
R. M. Yusif et al.: Some variables affecting the characteristics of Eudragit E-sodium alginate polyelectrolyte complex as a tablet matrix for diltiazem hydrochloride, Acta Pharm. 64 (2014) 89-104.

\section{Degree of tablet swelling}

In order to determine the degree of swelling, tablets of $185 \mathrm{mg}$ mass (polymer carrier) and $10 \mathrm{~mm}$ diameter were prepared by compressing the given amount of polymers, physical mixtures or the prepared PECs powder using a single punch tablet machine. The hardness of tablets used for the swelling study was in the range of (71.05-78.4 N) as DTZ HCl tablets. The degree of swelling was investigated under conditions that simulated the gastro-intestinal tract: the first $2 \mathrm{~h}$ in acidic medium $(\mathrm{pH} 1.2)$, and the next $6 \mathrm{~h}$ in a buffer solution ( $\mathrm{pH}$ 6.8). Compositions of the media used were those described in USPXXV as dissolution media No. 1 ( $\mathrm{pH} 1.2$ ) and No. 2 ( $\mathrm{pH}$ 6.8). The tablets were initially weighed, placed in a pre-weighed basket (from the dissolution test equipment) and weighed again, then immersed in $40 \mathrm{~mL}$ of swelling medium at $37.0 \pm 0.5^{\circ} \mathrm{C}$. After every hour, the basket was removed from the medium, accurately dried with filter paper, weighed and mass differences were determined. The degree of swelling $(\mathrm{S} \%)$ was calculated. The obtained results were the mean of three determinations.

\section{In vitro drug release studies}

The release of DTZ $\mathrm{HCl}$ from matrix tablets was determined using a six vessel dissolution tester complying with the USP requirements for Apparatus II (paddle) (DA-6D, India). A rotating speed of $100 \mathrm{rpm}$ and temperature of $37 \pm 0.5^{\circ} \mathrm{C}$ were applied in all experiments. The dissolution media used $(900 \mathrm{~mL})$ were those described in USPXXV as dissolution media No. 1 ( $\mathrm{pH}$ 1.2) for the first $2 \mathrm{~h}$ and No. 2 (pH 6.8) for subsequent $6 \mathrm{~h}$. Aliquots $(2 \mathrm{~mL})$ of solution were taken at specific time intervals and the volume was made up to the original value by adding fresh dissolution medium. The samples were diluted, filtered using a Millipore filter (Gelman GN-6 Metricel membrane filter, $0.45 \mu \mathrm{m}$ pore size and $47 \mathrm{~mm}$ diameter, USA) and the amounts of DTZ $\mathrm{HCl}$ released in the dissolution medium were determined spectrophotometrically at $236 \mathrm{~nm}$ against a calibration curve. Results were given as the mean values of three determinations. Preliminary experiments had shown that the polymers did not interfere with the model drug.

\section{Kinetics of drug release}

To characterize the drug release behavior from the polymeric systems and to understand the corresponding mechanism, the data of in vitro drug release from the prepared formulae were analyzed according to the Korsmeyer-Peppas semi-empirical model (17):

$$
M_{\mathrm{t}} / M_{\infty}=K t^{n}
$$

where $M_{\mathrm{t}} / M_{\infty}$ is the fraction of drug released at time $t, K$ is a constant incorporating the structural and geometric characteristics of matrix tablets, $n$ is the release exponent, indicative of the drug release mechanism; it is the slope of log fraction drug released versus log time.

\section{Statistical analysis}

The results were represented as mean $\pm \mathrm{SD}(n=3)$. Statistical significance of the differences in percent drug released was determined using one-way analysis of variance 
R. M. Yusif et al.: Some variables affecting the characteristics of Eudragit E-sodium alginate polyelectrolyte complex as a tablet matrix for diltiazem hydrochloride, Acta Pharm. 64 (2014) 89-104.

(ANOVA), followed by the Tukey-Kramer multiple comparison test at a level of significance of $p<0.05$ with Instat Graphpad prism software (version 4.0; Graphpad software, San Diego, California).

\section{RESULTS AND DISCUSSION}

\section{Viscosity measurements}

Eudragit $\mathrm{E}$ is soluble in acidic medium up to $\mathrm{pH} 6.0$ due to hydration of protonated dimethylamino groups. On the other hand, sodium alginate is soluble at $\mathrm{pH}$ above 2.5 due to ionization of carboxylic acid groups (18). The possibility of interaction between these two polyelectrolytes was investigated at two $\mathrm{pH}$ values, 4 and 5.8, in which both polymers were soluble and partially ionized.

Figure 1 shows how the supernatants viscosity changed when the EE/SA mass ratio changed. Viscosity progressively decreased with the increase in alginate concentration until reaching a minimum value at EE/SA mass ratio of 1.5:1, beyond which the viscosity started to increase. Minimum viscosity was a result of maximum interaction between the polymers, leading to the greatest consumption of free polymer molecules towards the formation of an insoluble complex and, hence, supernatants containing the least amount of dissolved or free polymers (15). It is also clear from Fig. 1 that alginate viscosity or $\mathrm{pH}$ of the media had no effect on the polymer interaction ratio. Similar results were obtained by Tapia et al. (7), who found that the optimal ratio between chitosan and SA was independent of the $\mathrm{pH}$ of dissolution media or the degree of polymerization of polymers used in the mixture. Awareness of the optimal interaction ratio of the complex is important during complex preparation to obtain a maximum complex yield. Furthermore, physical mixtures of EE and SA in tablets with the ratio 1.5:1 were prepared similarly to that of PEC for comparison.

Fig. 1. Supernatant viscosity from EE-SA systems as a function of $\mathrm{pH}$ of media and SA viscosity.

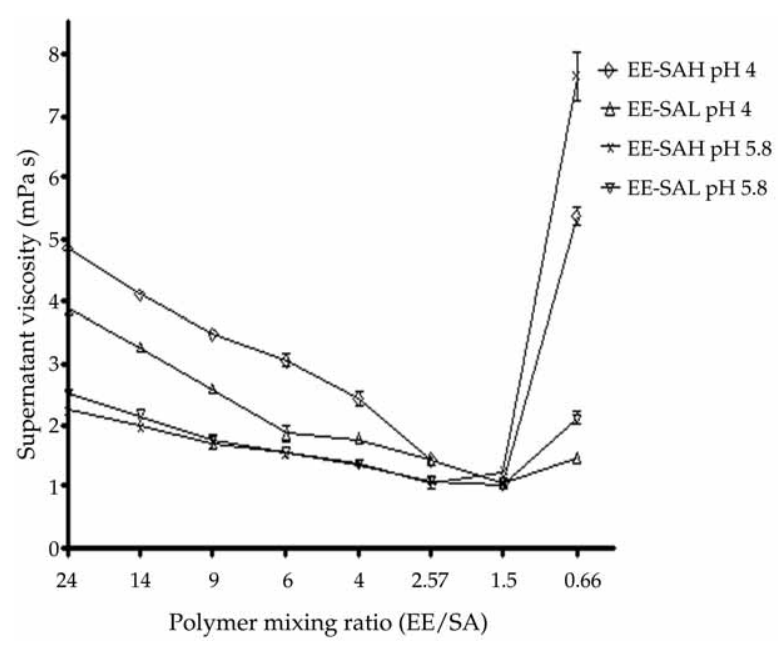


R. M. Yusif et al.: Some variables affecting the characteristics of Eudragit E-sodium alginate polyelectrolyte complex as a tablet matrix for diltiazem hydrochloride, Acta Pharm. 64 (2014) 89-104.

\section{Study of polymer-polymer and drug-polymer interaction}

Figure 2 shows the FT-IR spectra of EE, SAH, EE-SAH physical mixture and the corresponding PECs. The spectrum of EE showed a band characteristic of $\mathrm{C}=\mathrm{O}$ ester vibration at $1732 \mathrm{~cm}^{-1}$ as well as two bands at 2773 and $2821 \mathrm{~cm}^{-1}$, which can be assigned to the dimethylamino groups of EE (15). Sodium alginate spectrum showed absorption bands at 1612 and $1411 \mathrm{~cm}^{-1}$, which corresponded to asymmetric and symmetric stretching vibration of the carboxylate group (19). EE-SAH physical mixture showed characteristic bands of the carboxylate group of SA and dimethylamino group of EE. The FT-IR spectra of EE-SAH PEC obtained as precipitates in media with different $\mathrm{pH}$ values $(4$ and 5.8) were very different from those of EE, SAH alone or their physical mixture. The characteristic peak becoming more pronounced at 1466 and $1469 \mathrm{~cm}^{-1}$ for PEC at $\mathrm{pH} 4$ and 5.8, respectively, might be assigned to the absorption band of the carboxylate groups of SAH that formed ionic bonds with the protonated dimethylamino groups of EE. Also, two bands at 2480-2511 $\mathrm{cm}^{-1}$, for EE-SAH PEC at $\mathrm{pH} 4$ and at $2485-2513 \mathrm{~cm}^{-1}$ for EE-SAH PEC at pH 5.8, might be assigned to the absorption bands of polymer salts, which were brought about by the interaction of the dimethylamino groups of EE with the carboxyl groups of SAH. On the other hand, it can be clearly seen from Fig. 2 that EE-SAH PEC at pH 5.8 showed a characteristic band at $2820 \mathrm{~cm}^{-1}$, which was not found in EESAH PEC at $\mathrm{pH}$ 4. This means that these PEC samples had also non-ionized dimethyl-

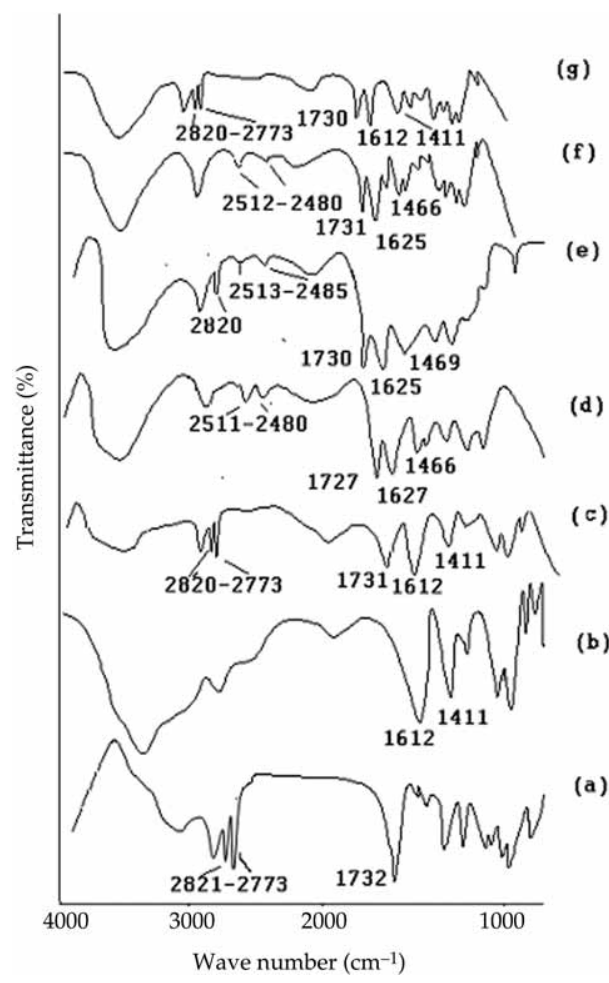

Fig. 2. FT-IR of: a) EE, b) SAH, c) EE-SAH physical mixture, d) EE-SAH PEC at $\mathrm{pH}$ 4, e) EE-SAH PEC at $\mathrm{pH} 5.8$, f) EE-SAH mixture kneaded with $0.1 \mathrm{~mol} \mathrm{~L}^{-1} \mathrm{HCl}$, and g) EE-SAH mixture kneaded with phosphate buffer of $\mathrm{pH} 6.8$. 
R. M. Yusif et al.: Some variables affecting the characteristics of Eudragit E-sodium alginate polyelectrolyte complex as a tablet matrix for diltiazem hydrochloride, Acta Pharm. 64 (2014) 89-104.

amino groups in their structure, since if the $\mathrm{pH}$ of the medium was increased, the number of protonated groups of EE was decreased, but they were still able to form PEC with SA molecules (18).

The EE-SAH physical mixture kneaded with 0.1 mol L ${ }^{-1} \mathrm{HCl}$ showed a spectrum similar to EE-SAH PEC at $\mathrm{pH}$ 4, while those kneaded with phosphate buffer of $\mathrm{pH} 6.8$ showed the characteristic bands of EE and SAH similar to that of the untreated physical mixture. Accordingly, it could be hypothesized that complexation between EE and SAH could occur within the tablet matrix during the dissolution process in $0.1 \mathrm{~mol} \mathrm{~L}^{-1} \mathrm{HCl}$, but not in phosphate buffer of $\mathrm{pH} 6.8$.

The DSC curves of EE, SAH, EE-SAH physical mixture and the prepared PECs obtained as precipitates in media with different $\mathrm{pH}$ values (4 and 5.8) are shown in Fig. 3. The thermogram of EE showed two endothermic peaks; one at 60.5 and the other at 300 ${ }^{\circ} \mathrm{C}$. The first endothermic peak was due to the glass transition temperature of EE, while the second one was attributed to the loss of water by condensation that resulted in the anhydride formation of EE $(15,20)$. The thermogram of SAH showed an initial endothermic peak at $87.6^{\circ} \mathrm{C}$ and an exothermic one at $251.5^{\circ} \mathrm{C}$. Endothermic peaks corresponded to the loss of water associated with the hydrophilic groups of polymers. While the exothermic peaks referred to the degradation of polyelectrolytes due to dehydration and depolymerization reactions most probably to the partial decarboxylation of the protonated carboxylic groups and oxidation reactions of the polyelectrolytes (19).

It can be seen from Fig. 3 that the peaks of complexes were shifted from those of the physical mixture. Peaks of the physical mixture appeared to be combinations of two polymers but they were different from those of PECs probably because complexation of polyelectrolytes resulted in new chemical bonds. The thermograms of PECs precipitated in media of different $\mathrm{pH}$ values (4 and 5.8) showed disappearance of the degradation

Fig. 3. DSC thermograms of: a) EE, b) $\mathrm{SAH}, \mathrm{c}) \mathrm{EE}-\mathrm{SAH}$ physical mixture, d) EE-SAH PEC at $\mathrm{pH} 4$, e) EE-SAH PEC at pH 5.8, f) EE-SAH mixture kneaded with $0.1 \mathrm{~mol} \mathrm{~L}^{-1}$ $\mathrm{HCl}$, and g) EE-SAH mixture kneaded with phosphate buffer of $\mathrm{pH}$ 6.8.

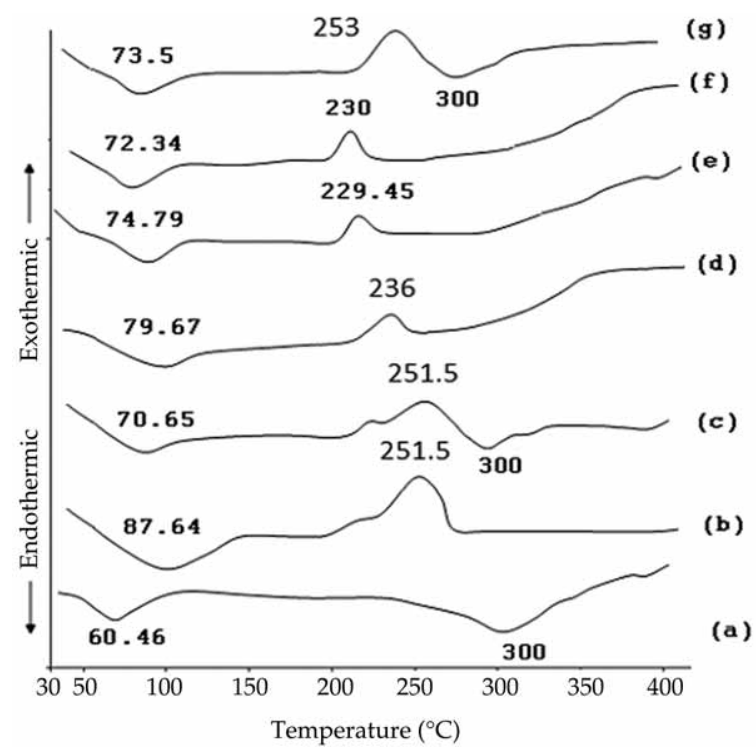


exothermic peak of SAH; however, new exothermic peaks appeared at 236 and $229.5^{\circ} \mathrm{C}$, respectively, which were assigned to the formation of an ionic pair between the carboxylate group of SAH and the dimethylamino group of EE. Similar exothermic peaks were found for the EE-carrageenan complex (15) that were related to PEC formation. On the other hand, the DSC thermogram of the EE-SAH mixture kneaded with $0.1 \mathrm{~mol} \mathrm{~L}^{-1}$ $\mathrm{HCl}$ showed a new exothermic peak at $230{ }^{\circ} \mathrm{C}$; however, the kneaded polymers in phosphate buffer at $\mathrm{pH} 6.8$ had the characteristic exothermic peak of $\mathrm{SAH}$ at $253{ }^{\circ} \mathrm{C}$ and the endothermic peak of $\mathrm{EE}$ at $300{ }^{\circ} \mathrm{C}$ similar to that of the untreated physical mixture. These findings agreed with the results obtained by FT-IR.

The interaction between EE and SAL was also studied and the results showed the same behavior as those of EE-SAH. Furthermore, the FT-IR spectra and DSC thermograms of DTZ HCl-polymers kneaded with $0.1 \mathrm{~mol} \mathrm{~L}^{-1} \mathrm{HCl}$ or phosphate buffer as well as the corresponding untreated physical mixtures reflected the characteristic features of DTZ $\mathrm{HCl}$ alone. This indicated the compatibility of DTZ $\mathrm{HCl}$ with the used polymers during the dissolution process (data not shown).

\section{Degree of tablet swelling}

The degree of tablet swelling is dependent on tablet hardness which influences the compaction of substances inside tablets and hence affects water penetration into the core (21). We therefore adjusted the compression pressure to maintain approximately constant hardness values.

The swelling behavior of tablets containing EE, SAH, EE-SAH PECs and the corresponding physical mixtures of different ratios are shown in Fig. 4. In the first stage of the experiment (acidic medium), the swelling degree could not be evaluated for the EE based matrix, which was dissolved within half an hour. This may be due to protonation of the dimethylamino groups of this polycation in the acidic medium (13). In contrast, tablets prepared from SAH did not disintegrate but swelled in acidic medium up to $115 \%$ after 2 h. At $\mathrm{pH}$ 1.2, SA rapidly converted to alginic acid, which is virtually insoluble and has the ability to swell on hydration with a tough and rubbery texture. After immersion in phosphate buffer of $\mathrm{pH} 6.8$, alginic acid converted to alginate and showed a higher degree of swelling than in acidic medium, which reached $236 \%$ after $3 \mathrm{~h}$. The tablet matrices containing SAH swelled and a viscous adhesive gel mass was formed when they came into contact with phosphate buffer of $\mathrm{pH}$ 6.8. Then, the produced gel layer started to dissolve at different rates and the \% swelling decreased until it reached $115 \%$ after $8 \mathrm{~h}$.

The swelling properties of tablets containing EE-SAH physical mixtures (PMH1-3) showed definitely different behavior (Fig. 4). They showed a slight increase in weight up to 65.4, 107 and $130 \%$ after $2 \mathrm{~h}$ in acidic medium for PMH1, PMH2 and PMH3, respectively. It is worth mentioning that the elevated micro-environmental $\mathrm{pH}$ provided by the dissolved EE in the swollen gel layer (22) supported the ionization of SAH within the tablet matrix and, hence, its interaction with EE. This was confirmed by dispersing amounts of EE equivalent to those found in the tablets $(45.5,109$ and $136.5 \mathrm{mg})$ in $1 \mathrm{~mL}$ of $0.1 \mathrm{~mol} \mathrm{~L}^{-1} \mathrm{HCl}(\mathrm{pH} 1.2)$ and by measurement of the $\mathrm{pH}$ of these solutions. The measured $\mathrm{pH}$ values were in the range 5.8-6.5. Accordingly, both polymers were sufficiently ionized for electrostatic interaction and consequently complex formation was enabled in 
Fig. 4. Swelling profiles of tablets containing EE-SAH PECs and their physical mixtures.

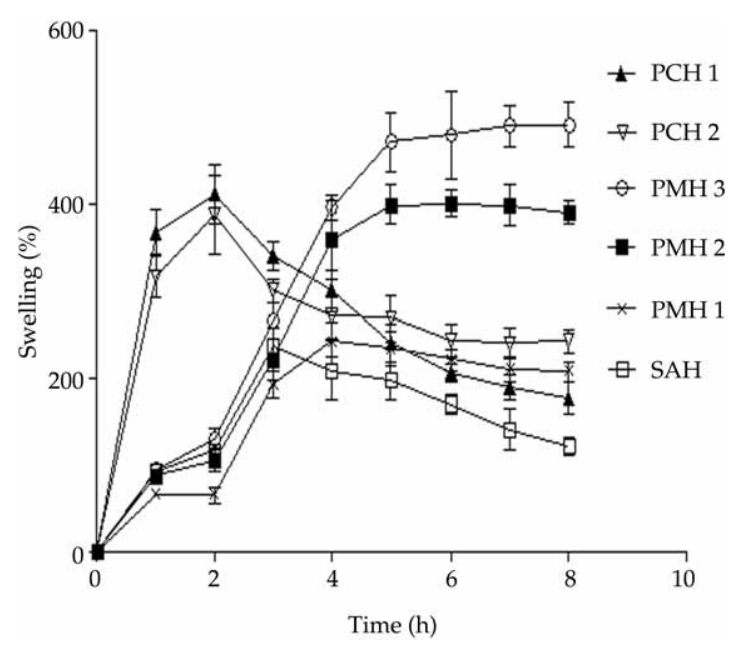

$0.1 \mathrm{~mol} \mathrm{~L}{ }^{-1} \mathrm{HCl}$ medium. Upon the passage to a buffer medium, the swelling continued to increase also by increasing the alginate ratio in the tablets up to the values of 233, 398 and $470 \%$ for PMH1, PMH2 and PMH3, respectively (at time $=5 \mathrm{~h}$ ). However, no further increase in swelling was observed after that and a plateau was reached. This can be explained by the fact that, at pH 6.8, the interaction between EE and SAH in the (PMH1-3) tablets was low because only SAH was ionized and EE was not. As a result, the increase in swelling ability of PMH1-3 tablets was due to the swelling of SAH. This behavior could be attributed to negligible ionic crosslinking between the polymers, so that no significant interpolymer interaction was present $(14,23)$.

On the other hand, it was found that all PMH tablets swelled more than SAH tablets alone. The higher the percent of SAH in the matrix, the higher was the percent swelling of the tablets, which effect was clearly observed in phosphate buffer medium compared to acidic medium. This may be due to the fact that dissolution or erosion of the SA gel layer formed around the tablets at $\mathrm{pH} 6.8$ was decreased by EE which was insoluble under high $\mathrm{pH}$ conditions.

Different behavior was observed for EE-SAH PECs in acidic medium, since they showed a higher degree of swelling than the corresponding physical mixtures (Fig. 4), where the degree of swelling increased to approximately 410 and $387 \%$ for PCH1 and $\mathrm{PCH} 2$, respectively, after the first $2 \mathrm{~h}$. This difference in swelling ability of the complexed polyelectrolyte (physical mixture and PEC) might be due to the change in structure of functional groups that had happened during continuous penetration of dissolution medium into the matrix (13). When the $\mathrm{pH}$ of the medium changed to 6.8 , the carboxylic acid groups of SAH were ionized. In contrast, protonation of the dimethylamino groups on EE was lost, resulting in a lower degree of interaction and thus allowing partial disintegration of EE-SAH PEC tablets (23). The obtained results have revealed that the main difference between PCH1-2 and PMH1-3 is the swelling rate and not maximum swelling. Nevertheless, PCH1-2 swelled faster in acidic medium, followed by a gradual decrease on immersion in phosphate buffer of $\mathrm{pH}$ 6.8, which might be due to erosion of 
R. M. Yusif et al.: Some variables affecting the characteristics of Eudragit E-sodium alginate polyelectrolyte complex as a tablet matrix for diltiazem hydrochloride, Acta Pharm. 64 (2014) 89-104.



Fig. 5. Swelling profiles of tablets containing EE-SAL PECs and their physical mixtures.

the tablets. In contrast, PMH1-3 tablets swelled slowly in acidic medium and thus required a higher $\mathrm{pH}$ (6.8) for increasing their swelling degree and most likely the swelling at this $\mathrm{pH}$ took place due to SAH swelling.

It was clearly observed from Fig. 5, that SAL tablets showed higher swelling than SAH tablets, which reached a value of $142 \%$ after $2 \mathrm{~h}$ in acid medium, indicating rapid hydration of SAL compared to SAH tablets. After $2 \mathrm{~h}$, the buffer stage began and the tablets started to dissolve; accordingly, the percent swelling continued to decrease until it reached $40 \%$ after $8 \mathrm{~h}$.

Tablets containing EE-SAL physical mixtures showed a higher degree of swelling than the corresponding EE-SAH tablets during the first $2 \mathrm{~h}$, where PML1, PML2 and PML3 increased by 121, 181.9 and $241.5 \%$, respectively. The increase in swelling corresponded to the amount of SAL in the tablets. A similar effect was previously observed with EE-SAH physical mixture matrices in acidic medium. After immersion of EE-SAL physical mixture tablets in phosphate buffer, a slower swelling rate was observed (Fig. 5). These results were likely due to rapid hydration in acid medium resulting in rapid complexation and gel formation at tablet surface during early times of swelling. This gel formation slowed down further solvent penetration inside the tablets and, hence, slower swelling afterwards. On the other hand, EE-SAL PEC tablets (PCL1 and PCL2) increased in weight by 200 and $197 \%$ after immersion for $2 \mathrm{~h}$ in the acid phase. In phosphate buffer, they showed a swelling behavior similar to that of the corresponding physical mixtures. It could be concluded from the swelling study that the degree of swelling was dependent on the type (SAH or SAL) and nature of the matrix of either the physical mixture or PECs. Regarding the nature, tablets containing matrices of EE-SAH physical mixtures showed a continuous and gradual increase in the swelling degree with a uniform shape and minimum disintegration compared to those prepared from EE-SAH PECs. On the other hand, tablets containing EE-SAL physical mixtures showed a swelling behavior similar to the corresponding PECs. In general, considerably higher maximum swelling was observed with SAH matrices. 
R. M. Yusif et al.: Some variables affecting the characteristics of Eudragit E-sodium alginate polyelectrolyte complex as a tablet matrix for diltiazem hydrochloride, Acta Pharm. 64 (2014) 89-104.

\section{In vitro drug release}

In $0.1 \mathrm{~mol} \mathrm{~L}^{-1} \mathrm{HCl}$, complete DTZ $\mathrm{HCl}$ release occurred within half an hour from tablets containing drug alone. Release of DTZ $\mathrm{HCl}$ from EE matrices was slow compared to tablets containing drug alone, and complete drug release was achieved within $2 \mathrm{~h}$ in $0.1 \mathrm{~mol} \mathrm{~L}^{-1} \mathrm{HCl}$ (Figs. 6 and 7). In contrast, sodium alginate is less soluble at acidic $\mathrm{pH}$ and the release of the drug from alginate tablets was the slowest compared to EE tablets or pure drug. Furthermore, the drug release from matrices containing SAH was slower compared to those of SAL (Figs. 6 and 7).

Upon changing the $\mathrm{pH}$ of the dissolution medium to 6.8 , drug release was still faster for SAL matrices ( $100 \%$ after $5 \mathrm{~h}$ ) compared to SAH matrices (60.5\% after $5 \mathrm{~h}$ ). It was reported that polymers with higher viscosities retarded drug release to a greater extent than lower viscosity polymers (24). The possible mechanism behind the rapid release rate produced by SAL matrices was rapid wetting during the dissolution test, forming a loose and porous outer gel layer that was easily eroded, resulting in a higher drug release rate due to enhanced mobility of macromolecules causing greater diffusivities of dissolution medium and drug (25).

Release profiles of DTZ HCl from tablets containing EE-SAH physical mixtures and the corresponding PECs are presented in Fig. 6. In the first $2 \mathrm{~h}$, at $\mathrm{pH} 1.2$, the percent drug released was 66.1, 50.6, 54.9, 41.2 and 38.5 from PCH1, PCH2, PMH1, PMH2 and PMH3 tablets, respectively. Drug release from PCH1 tablets was significantly higher $(p<$ 0.05) compared to PCH2 and PMH1-3 tablets, which appeared to be related to the high swelling degree of these tablets (26). Logically, diffusion of the drug was easier in more open swelled structures than that in more compact structures (27). Then, further increase in drug release from PCH1 was due to the erosion of tablets. Satoh et al. (28) reported that PEC has a hydrophobic nature, so that there might be a weakening of the interparticle binding force in the tablets when dissolution media penetrates into the tablets, resulting in rapid disintegration and, hence, increased drug release.

Fig. 6. Diltiazem hydrochloride release from tablets containing EE-SAH PECs and their physical mixtures.

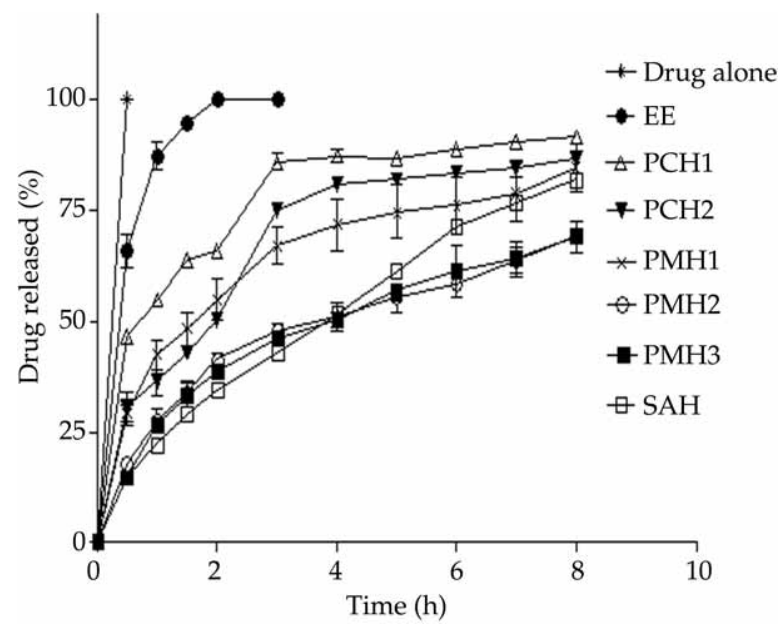




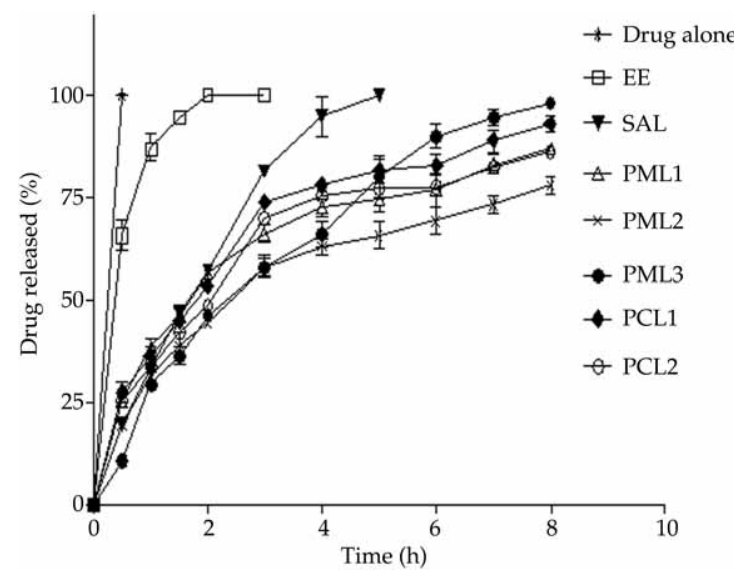

Fig. 7. Diltiazem hydrochloride release from tablets containing EE-SAL PECs and their physical mixtures.

When physical mixtures of EE-SAH, at weight ratios of 1.5:1 and 1:3 (PMH2 and PMH3), were used as matrices, they decreased drug release significantly $(p<0.05)$ compared to PCH1 and PCH2. This could be attributed to the formation of an insoluble PEC (gel-like layer), and consequently slower solvent penetration into the matrices and more controlled drug diffusion were achieved (15). However, increase of the EE-SAH mass ratio in the matrices to 3:1 resulted in increased drug release, which could be attributed to a less complex formation between the polymers and, hence, less control of solvent penetration and drug diffusion. In phosphate buffer of $\mathrm{pH} 6.8$, the percent drug released continued increasing after the first hour of exposure, then drug release was slowed down between 4 and $8 \mathrm{~h}$. The \% drug released after $8 \mathrm{~h}$ was 91.7, 86.9, 84.4, 69.2, and 68.9 from $\mathrm{PCH} 1, \mathrm{PCH} 2, \mathrm{PMH} 1, \mathrm{PMH} 2$ and $\mathrm{PMH} 3$, respectively.

Figure 7 illustrates that the percent drug released after $2 \mathrm{~h}$ in $0.1 \mathrm{~mol} \mathrm{~L}-1 \mathrm{HCl}$ from PCL1 tablets was significantly higher than from PCL2 tablets. Furthermore, changing the EE-SAL physical mixture ratio from 3:1 (PML1) to 1:3 (PML3) decreased the drug release significantly $(p<0.05)$ compared to tablets containing EE or SAL. This indicated that an interaction occurred between polymers during dissolution in acid medium. In phosphate buffer, the highest percent $(98 \%)$ drug released after $8 \mathrm{~h}$ was obtained from PML3 while the lowest drug release (78.2) was that from PML2 tablets. On the other hand, the percent drug released from PCL1, PCL2, and PML1 was 93, 86, and 87, respectively, which showed that the slowest drug release was obtained from PML2 tablets.

It could be observed from the drug release study that there was no significant difference between (PCH1 and PCL1) or (PCH2 and PCL2); thus, drug release from PEC tablets was dependent on the $\mathrm{pH}$ of complexation rather than on the viscosity of alginate. However, a significant difference between drug release from PMH2 and PML2 tablets was recorded, which may be contributed to increased viscosity of the gel produced from the former compared to the latter. This confirmed that alginate viscosity had a pronounced effect on drug release from tablets containing EE-SA physical mixtures in contrast to those containing PECs. 
R. M. Yusif et al.: Some variables affecting the characteristics of Eudragit E-sodium alginate polyelectrolyte complex as a tablet matrix for diltiazem hydrochloride, Acta Pharm. 64 (2014) 89-104.

\section{Kinetics of drug release}

It was previously reported that when a mixture of polymers was a matrix, accurate determination of the mechanism of drug release from this swelled matrix was complex. Its behavior as a prolonged drug release system was found to be dependent on the polymers' hydration, relaxation and gel-forming properties when the fluid got into the matrix (29). Thus, it was recommended to analyze the dissolution data using exponential models for these systems, such as the Korsmeyer-Peppas semi-empirical model. This model was not applied to EE tablets because of their rapid release in $0.1 \mathrm{~mol} \mathrm{~L}^{-1} \mathrm{HCl}$. Therefore, correlation coefficients and diffusional exponents for these formulations could not be calculated as a result of insufficient data points, up to $60 \%$, on the drug release profiles to provide accurate values. Other formulations gave $n$ values between 0.695 and 0.985 (i.e., $0.5<n<1$ ) indicating that the release mechanism of $\mathrm{DTZ} \mathrm{HCl}$ from these matrices was anomalous (non-Fickian), which suggested that both diffusion of the drug in the hydrated matrix and its erosion modulated drug release. Similar results were obtained in the release of DTZ HCl from polyvinyl alcohol-polyacrylic acid microspheres (30) and EE-carbopol PEC tablets (16).

\section{CONCLUSIONS}

The obtained results confirmed the importance of ionic interaction between oppositely charged EE and SA within the tablet matrix to control the release rate of DTZ HCl. The optimal interaction ratio between EE-SA was determined at 1.5:1 and was independent of the viscosity grade of SA and the $\mathrm{pH}$ of the medium in which the complex was obtained. FT-IR and DSC results confirmed the ionic interaction between EE and each of SAH and SAL in PECs. Also, this interaction was confirmed in the case of physical mixtures kneaded in $0.1 \mathrm{~mol} \mathrm{~L}^{-1} \mathrm{HCl}$, but not in phosphate buffer of $\mathrm{pH}$ 6.8. The swelling character and drug release profiles of EE-SA tablets showed their dependence on the type of SA either in PEC or physical mixture. EE-SAL matrices were less swellable and their PECs behaved similarly to physical mixtures. However, the affinity of EE-SAH physical mixtures and PEC matrices to acidic/neutral media was different and, consequently, their swelling behavior was different. Where water uptake by EE-SAH PECs tablets increased, this resulted in a higher swelling rate (not higher maximum swelling ability), followed by their disintegration and consequently faster drug release compared to their physical mixtures. Drug release from PEC tablets depended on the $\mathrm{pH}$ of complexation rather than on the viscosity of alginate. This may be a result of the $\mathrm{pH}$ -dependent solubility of the polymers (due to their chemical structure), which largely influenced the properties of polymer complexes and hence could be different from those of the initial components. Tablets prepared from EE-SA physical mixtures showed more sustained drug release compared to those containing PECs. This sustained release behavior depended on alginate viscosity, polymer ratios and the interaction between EE and SA in the physical mixture within the tablet matrix, which depended on the $\mathrm{pH}$ of dissolution media. Entry of dissolution medium into the tablets caused considerable structural changes to the matrix, which in turn affected the diffusional release of the drug. For example, the possible interaction between EE-SA within the tablet matrix dur- 
R. M. Yusif et al.: Some variables affecting the characteristics of Eudragit E-sodium alginate polyelectrolyte complex as a tablet matrix for diltiazem hydrochloride, Acta Pharm. 64 (2014) 89-104.

ing the dissolution process was suggested to sustain the release of DTZ $\mathrm{HCl}$ mainly by restricting and regulating the diffusion of the drug from such matrices better than from PECs tablets. Moreover, drug release from tablets containing EE-SA physical mixtures was retarded by an increase in alginate viscosity. However, alginate viscosity had no effect on the drug release from PEC matrices. In general, it is worth mentioning that the drug release in phosphate buffer of $\mathrm{pH} 6.8$ was slower than in acidic medium for both matrices (SAH and SAL).

\section{REFERENCES}

1. V. A. Kemenova, R. I. Moustafine, K. V. Alekseyev, A. M. Scorodinskaya, A. B. Zezin, A. I. Tencova and V. A. Kabanov, Applying interpolymer complexes in pharmacy, Pharmacia 60 (1991) 67-72.

2. E. Tsuchida and K. Abe, Interactions between macromolecules in solution and intermacromolecular complexes, Adv. Polym. Sci. 45 (1982) 1-147; DOI: 10.1007/BFb0017548.

3. B. Philipp, H. Dautzenberg, K. J. Linow, J. Koetz and W. Dawydoff, Polyelectrolyte complexes-recent developments and open problems, Progr. Polym. Sci. 14 (1989) 91-172; DOI: 10.1016/ 0079-6700(89)90018-X.

4. J. Koetz, H. Koepke, G. Schmidt-Naake and O. Vogl, Polyanion-polycation complex formation as a function of the position of the functional groups, Polymer 37 (1996) 2775-2781; DOI: 10.1016/ 0032-3861(96)87641-5.

5. S. Dumitriu and E. Chornet, Inclusion and release of proteins from polysaccharide-based polyion complexes, Adv. Drug Deliv. Rev. 31 (1998) 223-246; DOI: 10.1016/S0169-409X(97)00120-8.

6. G. Dhanuja, B. Smitha and S. Sridhar, Pervaporation of isopropanol-water mixtures through polyion complex membranes, Sep. Purif. Technol. 44 (2005) 130-138; DOI: 10.1016/j.seppur.2005. 01.001.

7. C. Tapia, E. Costa, M. Moris, J. Sapag-Hagar, F. Valenzuela and C. Basualto, Study of the influence of the $\mathrm{pH}$ media dissolution, degree of polymerization and degree of swelling of the polymers on the mechanism of release of diltiazem from matrices based on mixtures of chitosan/alginate, Drug Dev. Ind. Pharm. 28 (2002) 217-224; DOI: 10.1081/DDC-120002455.

8. R. K. Chang, Y. Peng, N. Trivedi and A. J. Shukla, Polymethacrylate, in Handbook of Pharmaceutical Excipients (Ed. R. C. Rowe, P. J. Sheskey and M. E Quinn), Pharmaceutical Press and American Pharmacists Association, USA 2009, pp. 525-533.

9. S. K. Bajpai and S. Shubhra, Investigation of swelling/degradation behaviour of alginate beads crosslinked with $\mathrm{Ca}^{2+}$ and $\mathrm{Ba}^{2+}$ ions, Reac. Func. Poly. 59 (2004) 129-140; DOI: 10.1016/ j.reactfunctpolym.2004.01.002.

10. S. Takka and F. Acartürk, Calcium alginate microparticles for oral administration: I-Effect of sodium alginate type on drug release and drug entrapment efficiency, J. Microencapsul. 16 (1999) 275-290; DOI: 10.1080/026520499289013.

11. C. V. Liew, L. W. Chan, A. L. Ching and P. W. Heng, Evaluation of sodium alginate as drug release modifier in matrix tablets, Int. J. Pharm. 309 (2006) 25-37; DOI: 0.1016/j.ijpharm.2005. 10.040 .

12. Martindale: The Complete Drug Reference (Ed. P. Kathleen), $35^{\text {th }}$ ed., The Pharmaceutical Press, London 2007, pp. 1136-1138.

13. R. I. Moustafine, I. M. Zaharov and V. A. Kemenova, Physicochemical and drug release properties of Eudragit ${ }^{\circledR} \mathrm{E}$ PO/Eudragit ${ }^{\circledR} \mathrm{L}$ 100-55 interpolyelectrolyte complexes, Eur. J. Pharmac. Biopharmac. 63 (2006) 26-36; DOI: 10.1016/j.ejpb.2005.10.005. 
R. M. Yusif et al.: Some variables affecting the characteristics of Eudragit E-sodium alginate polyelectrolyte complex as a tablet matrix for diltiazem hydrochloride, Acta Pharm. 64 (2014) 89-104.

14. H. J. Prado, M. C. Matulewicz, P. Bonelli and A. L. Cukierman, Basic butylated methacrylate copolymer/kappa-carrageenan interpolyelectrolyte complex: Preparation, characterization and drug release behaviour, Eur. J. Pharmac. Biopharmac. 70 (2008) 171-178; DOI: 10.1016/j.ejpb.2008.04.017.

15. A. Bani-Jaber, L. Al-Aani, H. Alkhatib and B. Al-Khalidi, Prolonged intragastric drug delivery mediated by Eudragit ${ }^{\circledR}$ E-carrageenan, polyelectrolyte matrix tablets, AAPS Pharm. Sci. Tech. 12 (2011) 354-361; DOI: 10.1208/s12249-011-9595-0.

16. A. H. Abd El-Gawad, E. M. Ramadan, O. A. Soliman and R. M. Yusif, Formulation and in-vivo study of diltiazem hydrochloride tablets prepared using interpolymer complexes, BJPR 2 (2012) 17-40.

17. R. W. Korsmeyer, R. Gurny, E. M. Doelker, P. Buri and N. A. Peppas, Mechanism of solute release from porous hydrophilic polymers, Int. J. Pharm. 15 (1983) 25-35; DOI: 10.1016/0378-5173 (83)90064-9.

18. R. I. Moustafine, V. A. Kemenova, and G. Van den Mooter, Characteristics of interpolyelectrolyte complexes of Eudragit E100 with sodium alginate, Int. J. Pharm. 294 (2005) 113-120; DOI: 10.1016/j.ijpharm.2005.01.029.

19. J. P. Soares, J. E. Santos, G. O. Chierice and ETG Cavalheiro, Thermal behavior of alginic acid and its sodium salt, Eclet Quim. 29 (2004) 57-64; DOI: 10.1590/S0100-46702004000200009.

20. S. Y. Lin, H. L. Yu and M. J. Li, Formation of six-membered cyclic anhydrides by thermally induced intramolecular ester condensation in Eudragit E film, Polymer 40 (1999) 3589-3593; DOI: 10.1016/S0032-3861(98)00488-1.

21. K. Saeio, Y. Pongpaibul, H. Vierstein and S. Okonogi, Factors influencing drug dissolution characteristic from hydrophilic polymer matrix tablet, Sci. Pharm. 75 (2007) 147-163; DOI: $10.3797 /$ scipharm.2007.75.147.

22. V. M. Rao, K. Engh and Y. Qiu, Design of $\mathrm{pH}$-independent controlled release matrix tablets for acidic drugs, Int. J. Pharm. 252 (2003) 81-86; DOI: 10.1016/S0378-5173(02)00622-1.

23. W. M. Obeidat, A. H. Abuznait and A. S. Sallam, Sustained release tablets containing soluble polymethacrylates: Comparison with tableted polymethacrylate IPEC polymers, AAPS Pharm. Sci. Tech. 11 (2010) 54-63; DOI: 10.1208/s12249-009-9348-5.

24. M. E. C. Aldrete and L. V. Robles, Influence of the viscosity grade and particle size of HPMC on metronidazole release from matrix tablets, Eur. J. Pharmac. Biopharmac. 43 (1997) 173-178; DOI: 10.1016/S0939-6411(96)00004-5.

25. J. Siepmann, H. Kranz, R. Bodmeier and N. A. Peppas, HPMC-matrices for controlled drug delivery: a new model combining diffusion, swelling and dissolution mechanisms and predicting the release kinetics, Pharm. Res. 16 (1999) 1748-1756; DOI: 10.1023/A:1018914301328.

26. C. Tapia, Z. Escobar, E. Costa, J. S. Hagar, F. Valenzuela, C. Basualto, M. N. Gai and M. Y. Pedram, Comparative studies on polyelectrolyte complexes and mixtures of chitosan-alginate and chitosan-carrageenan as prolonged diltiazem chlorhydrate release systems, Eur. J. Pharmac. Biopharmac. 57 (2004) 65-75; DOI: 10.1016/S0939-6411(03)00153-X.

27. H. M. Fahmy and M. M. G. Fouda, Crosslinking of alginic acid/chitosan matrices using polycarboxylic acids and their utilization for sodium diclofenac release, Carbohyd. Polym. 73 (2008) 606-611; DOI: 10.1016/j.carbpol.2007.12.024.

28. K. Satoh, K. Takayama, Y. Machida, Y. Suzuki and T. Nagai, Disintegration and dissolution characteristics of compressed tablets consisting of hydroxypropyl cellulose and carboxyvinyl polymer, Chem. Pharm. Bull. 37 (1989) 1642-1644.

29. M. M. Talukdar, I. Vinckier, P. Moldenaers and R. Kinget, Rheological characterization of xanthan gum and hydroxypropylmethyl cellulose with respect to controlled-release drug delivery, J. Pharm. Sci. 85 (1996) 537-540; DOI: 10.1021/js950476u.

30. D. Ray, P. S. Gils, G. P. Mohanta, R. Manavalan and P. K. Sahoo, Comparative delivery of diltiazem hydrochloride through synthesized polymer. Hydrogel and hydrogel microspheres, J. Appl. Polym. Sci. 116 (2010) 959-968; DOI: 10.1002/app.31661. 